\title{
Revisión de literatura sobre modelamiento y simulación de fenómenos sociotecnológicos mediante minería de datos en bases de datos académicas*
}

\author{
Jheimer Julian Sepúlveda Lopez, \\ Doctor en Ingeniería, Universidad Nacional de \\ Colombia. Magíster en Administración, Universidad \\ Nacional de Colombia. Administrador de sistemas \\ informáticos, Universidad Nacional de Colombia. \\ Docente investigador, Universidad Nacional Abierta y a \\ Distancia (UNAD).jheimer.sepulveda@unad.edu.co \\ https://orcid.org/0000-0001-7632-3197
}

\section{LuzArabany Ramírez Castañeda,}

Doctora en Sostenibilidad, Tecnología y Humanismo, Universidad Politécnica de Cataluña-España.

Magíster en Medio Ambiente y Desarrollo, Universidad

Nacional de Colombia. Ingeniera de sistemas,

Universidad Autónoma de Manizales. Profesora

asociada, Facultad de Administración, Departamento de Informática y Computación, Universidad Nacional de

Colombia, sede Manizales

laramirezc@unal.edu.co

https://orcid.org/0000-0003-1635-2396

\section{Diana Patricia Bautista Sáenz}

Administradora de sistemas informáticos, Universidad Nacional de Colombia. Estudiante de Maestría en

Administración de Sistemas Informáticos, Universidad

Nacional de Colombia, sede Manizales.

dpbautistas@unal.edu.co

https://orcid.org/0000-0003-4400-0697

\section{Alexander Marin Florez}

Bibliotecólogo, Universidad de Antioquia, Escuela Interamericana de Bibliotecología. Unidad de Análisis Estratégico de Información, Sección de Bibliotecas, Universidad Nacional de Colombia, sede Manizales. almarinfl@unal.edu.co

https://orcid.org/0000-0002-6236-2934

\section{Sandra Milena Arredondo Lopez}

Especialista en Gestión Cultural con Énfasis en planeación y políticas culturales, Universidad Nacional de Colombia. Profesional en Ciencias de la Información, Bibliotecología y Archivística, Universidad del Quindío Unidad de Análisis Estratégico de Información, Sección de Bibliotecas, Universidad Nacional de Colombia, sede Manizales.smarredondol@unal.edu.co https://orcid.org/0000-0001-8319-2367

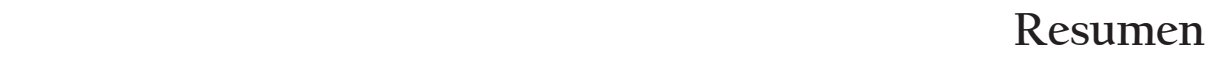

Los fenómenos sociotecnológicos se caracterizan por integrar aspectos sociales, técnicos y tecnológicos. Este tipo de fenómenos están compuestos por personas que pretenden alcanzar una serie de objetivos por medio de herramientas tecnológicas. Para entender el comportamiento dinámico de estos fenómenos, se hace necesario realizar procesos de modelamiento y simulación que integren los múltiples elementos y relaciones que los componen. El objetivo de este documento fue explicar el campo teórico del modelamiento y la simulación de los fenómenos sociotecnológicos desde un proceso de revisión sistemática de la literatura, la aplicación de la metodología de vigilancia tecnológica UNE 166006:201l y minería de datos mediante el software Vantage Point. Como una de las conclusiones identi icadas se menciona que este campo está abierto a la investigación que desde Colombia y Latinoamérica se pueda hacer.

Palabras clave: revisión de literatura, modelamiento, simulación, minería de datos, fenómeno sociotecnológico.

Cómo citar este artículo: Sepúlveda, Jheimer; Ramírez, Luz; Bautista, Diana; Marín, Alexander; Arredondo, Sandra (2021). Revisión de literatura sobre modelamiento y simulación de fenómenos sociotecnológicos mediante minería de datos en bases de datos académicas. Revista Interamericana de Bibliotecología, 44(2), e339215. https://doi.org/10.17533/udea.rib.v44n2e339215

Recibido: 2019-07-22 / Aceptado: 2021-04-23 


\section{Review of Literature on Modeling and Simulation of Socio-Technological Phenomena Through data Mining in Academic Databases}

\begin{abstract}
The socio-technological phenomena are characterized by integrating social, technical, and technological aspects. This type of phenomena are composed of people who intend to achieve a series of objectives through technological tools. To understand the dynamic behavior of these phenomena, it is necessary to carry out modeling and simulation processes that integrate the multiple elements and relationships that compose them. The objective of this document is to explain the theoretical field of modeling and the simulation of sociotechnological phenomena from a systematic literature review process, the application of UNE 166006: 2011 technology monitoring methodology, and data mining using Vantage software. Point. As one of the conclusions identified, it is mentioned that this field is open to research that can be done from Colombia and Latin America.
\end{abstract}

Keywords: Literature review, modeling, simulation, data mining, socio-technological phenomenon.

\section{Introducción}

Los fenómenos sociotecnológicos se caracterizan por integrar aspectos sociales, técnicos y tecnológicos; están compuestos por personas que pretenden alcanzar una serie de objetivos por medio de la utilización de herramientas tecnológicas. La coordinación entre lo social, técnico y tecnológico es fundamental y la gestión de los diversos procesos que los componen se convierte en una tarea que requiere de diferentes puntos de vista y su integración para comprender su complejidad. Para entender el comportamiento dinámico de estos fenómenos se hace necesario realizar procesos de modelamiento y simulación que integren los múltiples elementos y relaciones que los componen; un modelo permite tener una representación de un sistema a partir de sus elementos y las relaciones que a su vez conforman su estructura. Por su parte, la simulación permite obtener conocimiento de un sistema a través de sus cambios en el tiempo y entender su comportamiento y establecer posibles cambios en el futuro.

Con base en lo anterior, se estableció como objetivo de este estudio realizar una revisión de literatura en bases de datos académicas sobre propuestas de modelamiento y simulación de fenómenos sociotecnológicos y usar minería de datos para describir esta área de trabajo. Este estudio se enmarca en un trabajo final de maestría en Administración de Sistemas Informáticos de la Universidad Nacional de Colombia. El documento tiene la siguiente estructura: primero, se muestra lo que se entiende por un fenómeno sociotecnológico; segundo, se justifica la necesidad de realizar modelamiento y simulación de este tipo de fenómenos; tercero, se muestra el proceso de búsqueda sistemática realizado; cuarto, se muestran los resultados obtenidos; y quinto, se enuncian las conclusiones.

\section{2. ¿Qué es un fenómeno sociotecnológico?}

El concepto de sistema sociotécnico se estableció para enfatizar la interrelación recíproca entre humanos y máquinas, y para fomentar la configuración de las condiciones técnicas y sociales del trabajo, de tal manera que la eficiencia y la humanidad ya no se contradigan entre sí (Ropohl, 1999). Un sistema sociotécnico (STS) consiste en una interacción de humanos, organizaciones y sistemas técnicos. Los STS son heterogéneos, dinámicos, impredecibles y débilmente controlables (Fabiano-Dalpiaz \& Mylopoulos, 2011).

Para Lee (2006), las siguientes son las características de los fenómenos sociotecnológicos:

- Tienen propiedades emergentes que dependen de los componentes del sistema y sus relaciones.

- Son fenómenos no deterministas, ya que no siempre producen el mismo resultado cuando se tienen las mismas entradas, porque el comportamiento de los sistemas es parcialmente dependiente de los operadores humanos.

Existe una serie de fenómenos sociotecnológicos que se caracterizan por integrar aspectos sociales y técnicos o tecnológicos; a continuación, se relacionan diferentes planteamientos sobre estos fenómenos: 
- "Los sistemas sociotécnicos modernos se caracterizan por una gran complejidad estructural y conductual. Estos sistemas incluyen componentes de hardware y software con diferentes grados de autonomía y complejidad que interactúan y permiten la interacción entre los seres humanos" (Sharpanskykh, 2011, p. 308).

- Mumford (2006, citado en Bider \& Otto, 2015) menciona que "se considera que un sistema socio-técnico consiste en personas organizadas de alguna manera y utilizando ciertas técnicas y tecnología para asegurar el funcionamiento del sistema" (p. 1).

- El modelamiento y análisis de los sistemas sociotécnicos modernos es una tarea difícil (Davidsson, 2000, p. 102), ya que involucra variables cuantitativas y cualitativas.

- Para Ferscha, Zia, Riener y Sharpanskykh (2011), "al modelar (y simular) un sistema socio-tecnológico el enfoque primario ha estado en el lado tecnológico casi ignorando la dimensión social del mismo" (p. 235).

Con base en lo anterior, se concluye que los fenómenos sociotecnológicos son aquellos en los cuales el funcionamiento de un sistema o el cumplimiento de ciertos objetivos dependen del uso y apropiación de herramientas tecnológicas por parte de las personas.

\section{Modelamiento y simulación de}

\section{fenómenos sociotecnológicos}

Se ha identificado que, para comprender el aspecto dinámico de los fenómenos sociotecnológicos, es necesario realizar procesos de modelamiento y simulación que permitan comprender su estructura y comportamiento. Por tal motivo, se aclara lo que se entiende por modelamiento y por simulación.

Para EcuRed (2017), la simulación

es la imitación del funcionamiento de un sistema real durante un intervalo de tiempo. Esta simulación puede realizarse ya sea de forma manual o computacional.
La simulación se basa en un modelo de la realidad que cuenta una historia y al observar el comportamiento de esta, nos permite obtener conocimiento acerca del sistema real. La simulación permite el estudio y experimentación con las interacciones internas de un sistema complejo y ver los efectos que un cambio produce en el comportamiento de un sistema. (p. l)

Por otra parte, UTADEO (2017) menciona que

un modelo puede ser entendido como una representación, bien sea abstracta, análoga, fenomenológica o idealizada, de un objeto que puede ser real o ficticio. Mediante el modelado se busca mejorar el conocimiento y la comprensión de un fenómeno o proceso y ello involucra el estudio de la interacción entre las partes de un sistema y el sistema como un todo. La efectividad de los modelos como herramientas de certidumbre científica o como instrumentos de garantía para tomar decisiones sobre procesos de ingeniería, depende del nivel de correspondencia que pueda lograrse, dentro de los rangos relevantes, entre el modelo y el sistema real representado; entre el comportamiento observado al operar el modelo y el comportamiento observado de la experimentación sobre el sistema real. (p. l)

Por lo anterior, modelar es definir o describir la estructura del sistema; simular es tomar un modelo y estudiar su comportamiento en el tiempo. A continuación, se muestra la relevancia de modelar y simular los fenómenos sociotecnológicos.

Ferscha et al. (2011) mencionan que

el modelado de un sistema socio-técnico comienza con el modelado de entidades representativas individuales que son esencialmente heterogéneas, apareciendo a gran escala real y requiriendo numerosas interacciones a nivel individual. Por lo tanto, es difícil modelar un sistema de este tipo a nivel variable (usando ecuaciones estructurales) o sistema (usando ecuaciones diferenciales). (p. 235)

En su trabajo afirman que el modelamiento basado en agentes ha ganado popularidad, pero ellos contribuyen con un modelo de agente cognitivo. Por otro lado, plantean que su modelo 
es un modelo de toma de decisiones afectivo general de los procesos cognitivos de un agente relacionado con los atributos cognitivos afectados y que influyen en una situación de evacuación. Estos atributos son: (i) Intenciones: Confianza hacia los agentes vecinos y Creencia por las opciones (salidas), (ii) Emociones: Miedo por las opciones, Esperanza por las opciones y la atracción resultante por las opciones, y (iii) Individualismo: Expresividad, apertura y contagio de agentes. (p. 236)

Sharpanskykh (2011) utiliza el modelamiento basado en agentes como herramienta o modelo para la simulación de sistemas sociotecnológicos. Este autor afirma que

los modelos de sistemas socio-técnicos basados en agentes deberían explicar la complejidad del comportamiento humano, la heterogeneidad y el comportamiento autónomo de los sistemas técnicos, así como la interacción entre todos los actores del sistema. Se requieren modelar tanto las propiedades del sistema cuantitativas como cualitativas. (p. 308)

Dignum y Tan (2011) proponen un metamodelo para sistemas de modelo multiagente (MASQ META-MODEL Multi-agent), en el cual realizan

una caracterización de un dominio como una descomposición a lo largo de dos ejes: las perspectivas individuales frente a colectivas de un lado y las perspectivas interiores (es decir, estados mentales, representaciones) frente a exteriores (es decir, comportamiento, objetos, organizaciones) de otro lado. (p. 63)

Hassannezhad, Cantamessa y Montagna (2015) proponen "una herramienta de modelado, al establecer un equilibrio entre la naturaleza detallada del rigor de los modelos dinámicos de tareas y la flexibilidad y abstracción de los modelos de redes sociales, denominada 'señalización basada en actores' (ABS)" (p. 1).

Georgas y Sarma (2011) presentan el lenguaje STCML, destinado a apoyar el modelado de los aspectos sociotécnicos. "STCML tiene como objetivo proporcionar soporte, actualmente ausente de otras herramientas y enfoques, para especificar las relaciones entre los artefactos arquitectónicos y los procesos sociales" (p. 61).
Se concluye que los procesos de modelamiento y simulación permiten considerar los múltiples y diversos elementos y relaciones que componen los fenómenos sociotecnológicos. Permiten además analizar el comportamiento autónomo de los sistemas tecnológicos en combinación con el comportamiento humano; se debe considerar que estos participan como actores de los procesos.

\section{Búsqueda sistemática sobre mode- lamiento y simulación de fenómenos sociotecnológicos}

Las revisiones sistemáticas como un tipo de investigación científica que tiene como propósito integrar de forma objetiva y sistemática los resultados de los estudios empíricos sobre un determinado problema de investigación, con objeto de determinar el "estado del arte" en ese campo de estudio (Sánchez-Meca, 2010). Uno de los factores fundamentales en un proceso de revisión sistemática de literatura es la definición del proceso de búsqueda y con ello las fuentes que se consultaron (Galvis \& Sánchez, 2014), asimismo, el otro factor fundamental es la definición de la ecuación de búsqueda. A continuación, se enuncia el proceso realizado para la construcción de ese documento.

\subsection{Estrategia de búsqueda}

Con el propósito de describir la temática sobre modelamiento y simulación de fenómenos sociotecnológicos, se realizó un proceso de revisión de literatura y minería de datos apoyado en la herramienta Vantage Point.

Para cumplir este objetivo, se plantearon tres preguntas de interés:

1. ¿Cuáles son los métodos de simulación y modelamiento utilizados para abordar fenómenos sociotecnológicos?

2 ¿Qué herramientas y métodos se utilizan para hacer simulación o modelamiento de inclusión digital?

3 ¿Qué herramientas y métodos se utilizan para hacer simulación o modelamiento de ciudades inteligentes? 
Se incluye en las preguntas la ciudad inteligente y la inclusión digital como fenómenos sociotécnicos, ya que hacen parte de proyectos de investigación que se desarrolla actualmente en el grupo de investigación al cual pertenece el trabajo final de maestría en Administración de Sistemas Informáticos, en el cual se enmarca este proceso de revisión.

Con estas preguntas se procedió a construir la estrategia de búsqueda que tiene cuatro componentes (Tabla 1). El primer componente hace referencia al objetivo de la búsqueda, los otros tres componentes atienden a las perspectivas de los fenómenos sociotecnológicos y al área de aplicación.

Se realizó la búsqueda de material bibliográfico en las bases de datos académicas Scopus y Web of Scien- ce (WoS); igualmente, se utilizó el software Vantage Point para analizar indicadores bibliométricos mediante minería de datos sobre los metadatos de los resultados obtenidos. Antes de iniciar el proceso de diseño y construcción de informes, los resultados exportados de cada base de datos debieron ser refinados y organizados; estos procesos consisten en la revisión de los diferentes metadatos y sus significados, y la revisión y unificación de los procesos de transformación de los datos de título y resumen a lenguaje natural.

\subsection{Bases de datos}

Las ecuaciones de búsqueda que se construyeron con base en esta estrategia y que se aplicaron en las bases de datos Scopus y WoS en el mes de noviembre de 2017 se muestran a continuación:

Tabla l. Estrategia de búsquedas minería Vantage Point

\begin{tabular}{|c|c|c|c|}
\hline Objetivo & Perspectiva l & Perspectiva 2 & Perspectiva 3/Área de aplicación \\
\hline \multirow{9}{*}{$\begin{array}{l}\text { Models } \\
\text { Mode?ing }\end{array}$} & & & "Smart City" \\
\hline & & & "Smart cities" \\
\hline & Socio & Technological & "Intelligent city" \\
\hline & Social & Techn* & "Digital City" \\
\hline & Community & ICT & "City Planning" \\
\hline & & & "Connected Communities" \\
\hline & & & "Sustainable cities" \\
\hline & & & "Digital Inclusion" \\
\hline & "Social Inequality" & & \\
\hline Simulation & & & "Digital Gap" \\
\hline Simulators & "Social Welfare" & & "Digital Divide" \\
\hline & social benaviour & & "Technological Gap" \\
\hline "Simulation models" & & & \\
\hline "Simulation Techniques" & & & \\
\hline "Computer Simulation" & & & \\
\hline
\end{tabular}

Fuente: elaboración propia. 
4.2.1 Scopus

TITLE-ABS-KEY (models OR mode?ing OR simulation OR simulators OR "Simulation models" OR "Simulation Techniques" OR "Computer Simulation" ) AND TITLE-ABS-KEY ( socio OR social OR community OR "Social Inequality" OR "Social Welfare" OR "Social Behaviour" ) AND TITLE-ABS-KEY ( technological OR techn* OR ict) AND TITLE-ABS-KEY ( "Smart City" OR "Smart cities" OR "Intelligent city" OR "Digital City" OR "City Planning" OR "Connected Communities" OR "Sustainable cities" OR "Digital Inclusion" OR "Digital Gap" OR "Digital Divide" OR "Technological Gap" )

\subsubsection{Web of Science}

ts=(models OR mode?ing OR simulation OR simulators OR "simulation models" OR "simulation techniques" OR "computer simulation") AND ts=(socio OR social OR community OR "social inequality" OR "social welfare" OR "social behaviour") AND ts=(technological OR techn* OR ICT) AND ts=("smart city" OR "smart cities" OR "intelligent city" OR "digital city" OR "city planning" OR "connected communities" OR "sustainable cities" OR "digital inclusion" OR "digital gap" OR "digital divide" OR "technological gap")
Los periodos de tiempo para las dos búsquedas fueron 2003 a 2017 y se realizó búsqueda con base en los índices SCI-EXPANDED, SSCI, A\&EHCI y ESCI.

\subsection{Resultados de búsqueda}

Los resultados de este proceso de búsqueda se muestran en la Tabla 2.

Tabla 2. Resultados búsqueda de información para Vantage Point

\begin{tabular}{lc}
\hline \multicolumn{1}{c}{ Fuente } & Resultados \\
\hline Scopus & 809 \\
WoS & 1328 \\
Total & 2137 \\
\hline
\end{tabular}

Fuente: elaboración propia.

\section{Análisis de resultados}

La primera revisión que se hace sobre los resultados obtenidos es sobre la producción académica en el tema (Figura 1). Este proceso tuvo un avance significativo entre los años 2003 y 2005, un leve retroceso entre los años 2005 y 2007, a partir del 2007 se estabiliza el crecimiento, a partir del año 2012 se produce un crecimiento exponencial de material sobre el tema, el cual se mantiene estable hasta el año 2015, cuando nuevamente tiene un retroceso a partir del cual se ha generado una segunda fase en su crecimiento exponencial hasta el año 2016, manteniéndose linealmente en el año 2017

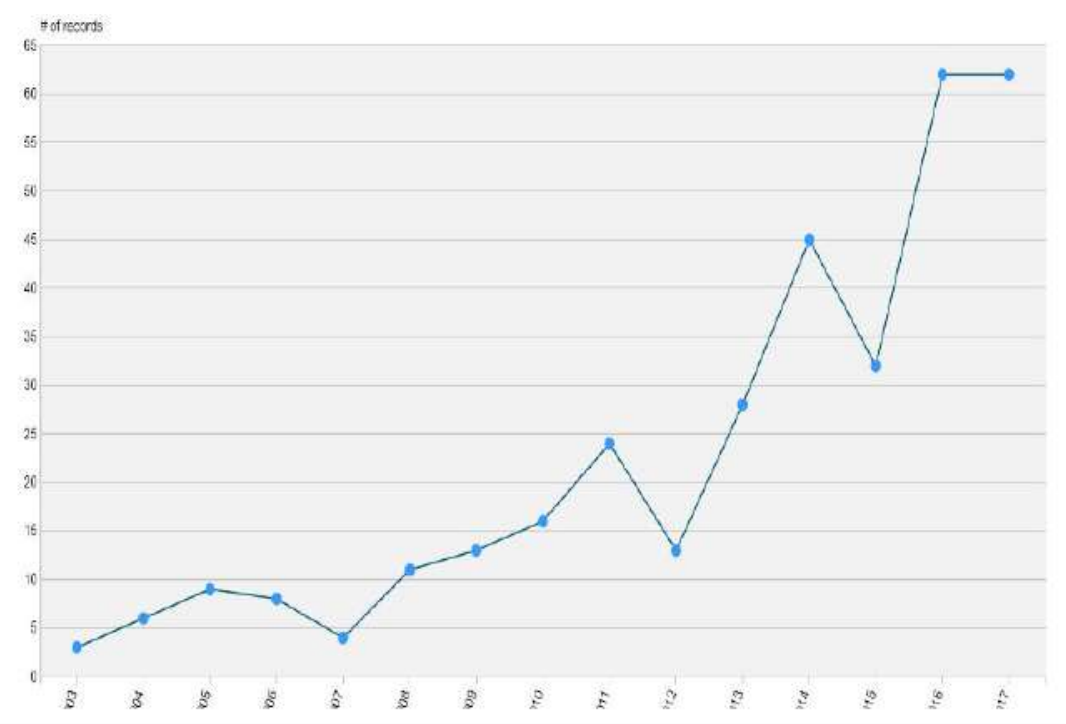

Figura l. Evolución del tema investigativo por años.

Fuente: creación propia a partir de Vantage Point, fusión de registros de WoS y Scopus, porcentaje de cubrimiento 100 \%. 
En la Figura 2 se muestra un mapa de calor con la producción académica por regiones sobre modelamiento y simulación de fenómenos sociotecnológicos a nivel mundial. El país con mayor cantidad de registros es Estados Unidos, seguido de Italia y del Reino Unido. Un aspecto para resaltar es que no se encontró ninguna producción académica realizada en Colombia.
En la Figura 3 se listan las instituciones que investigan en el tema y el periodo de publicación de material bibliográfico. Se muestra cómo la investigación realizada sobre estos temas específicos en las diferentes instituciones se ha desarrollado desde el año 2005 hasta la actualidad de forma constante.

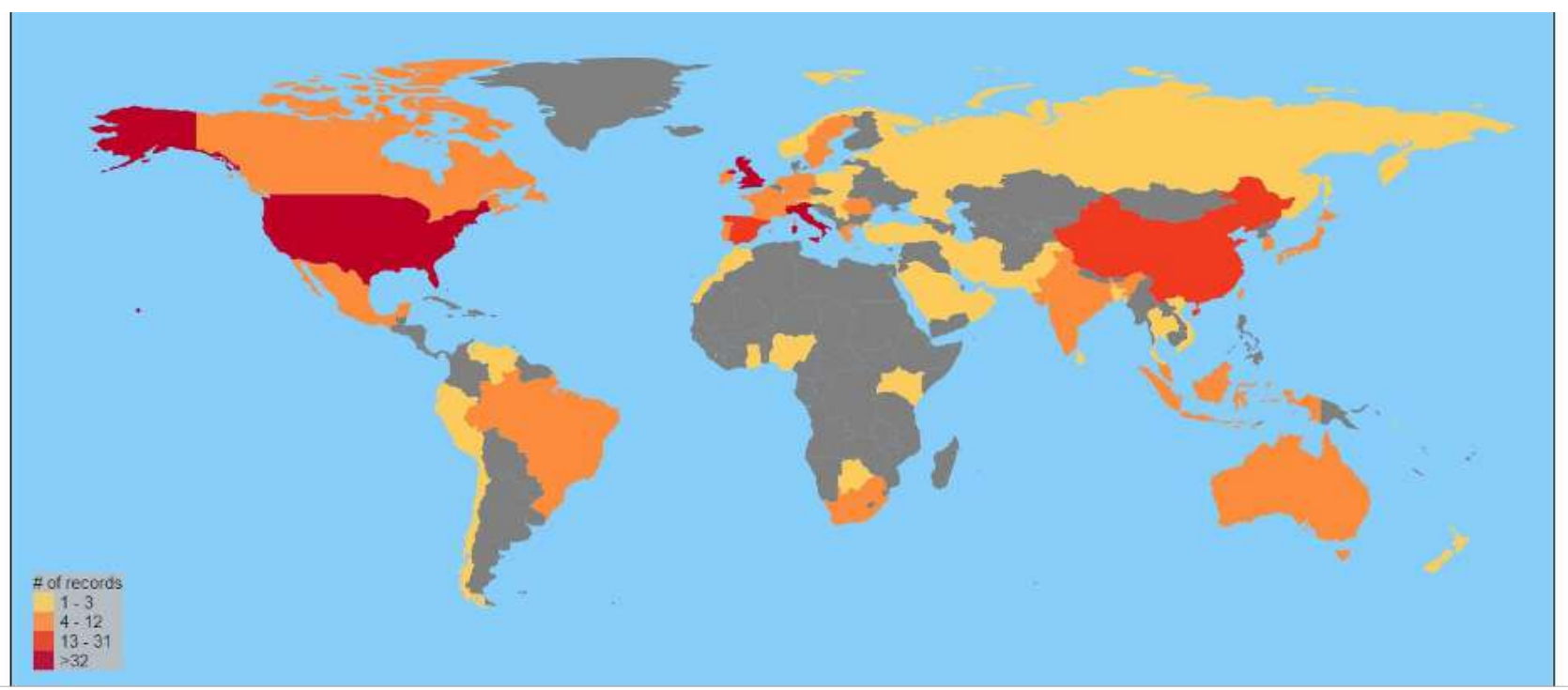

Figura 2. Mapa de calor de la producción científica a nivel mundial.

Fuente: creación propia a partir de Vantage Point, fusión registros WoS y Scopus, porcentaje de cubrimiento $96 \%$.

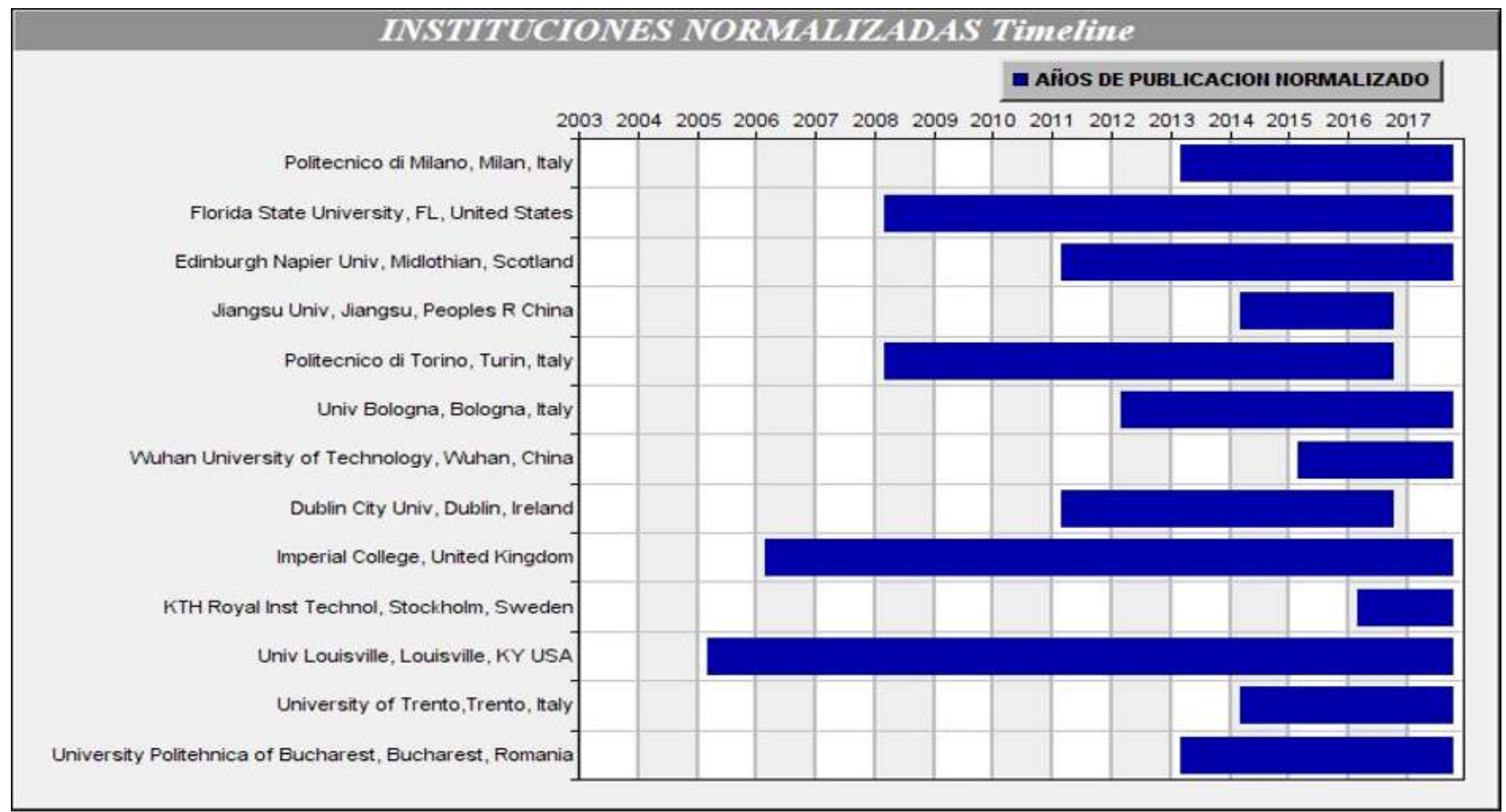

Figura 3. Instituciones que investigan en el tema y su frecuencia en el tiempo.

Fuente: creación propia a partir de Vantage Point, fusión de registros de WoS y Scopus, porcentaje de cubrimiento 96 \%. 
En la Figura 4 se muestra la clasificación de los países con mayor cantidad de producción académica sobre modelamiento y simulación de fenómenos sociotecnológicos y la colaboración existente entre ellos. Aunque a primera vista parece que existe interacción entre los países, las colaboraciones son mínimas comparadas con la cantidad de documentos académicos que produce cada uno a nivel individual. Según el mapa, los países con mayor cantidad de publicaciones son Estados Unidos, Italia, Reino Unido y China.

Para especificar más la colaboración entre estos países, en la Tabla 3 se muestra la producción de cada uno de ellos y el porcentaje de participación.
En la Figura 5 se muestra una nube de las palabras clave más utilizadas por autores en el campo de interés.

Esta nube permite identificar que la búsqueda realizada cumple con las expectativas y que la inclusión digital y las ciudades inteligentes se configuran como fenómenos sociotecnológicos. De igual manera, se puede identificar que, entre los elementos a considerar, se encuentran de forma reiterada las tecnologías, los aspectos sociales, los temas urbanos y sustentables.

Por último, en la Figura 6 se enuncian las diferentes herramientas de modelamiento y simulación de fenómenos sociotecnológicos más utilizadas. Se logró identificar que estas herramientas son las redes complejas, los sistemas multiagente o simulación basada en agentes.

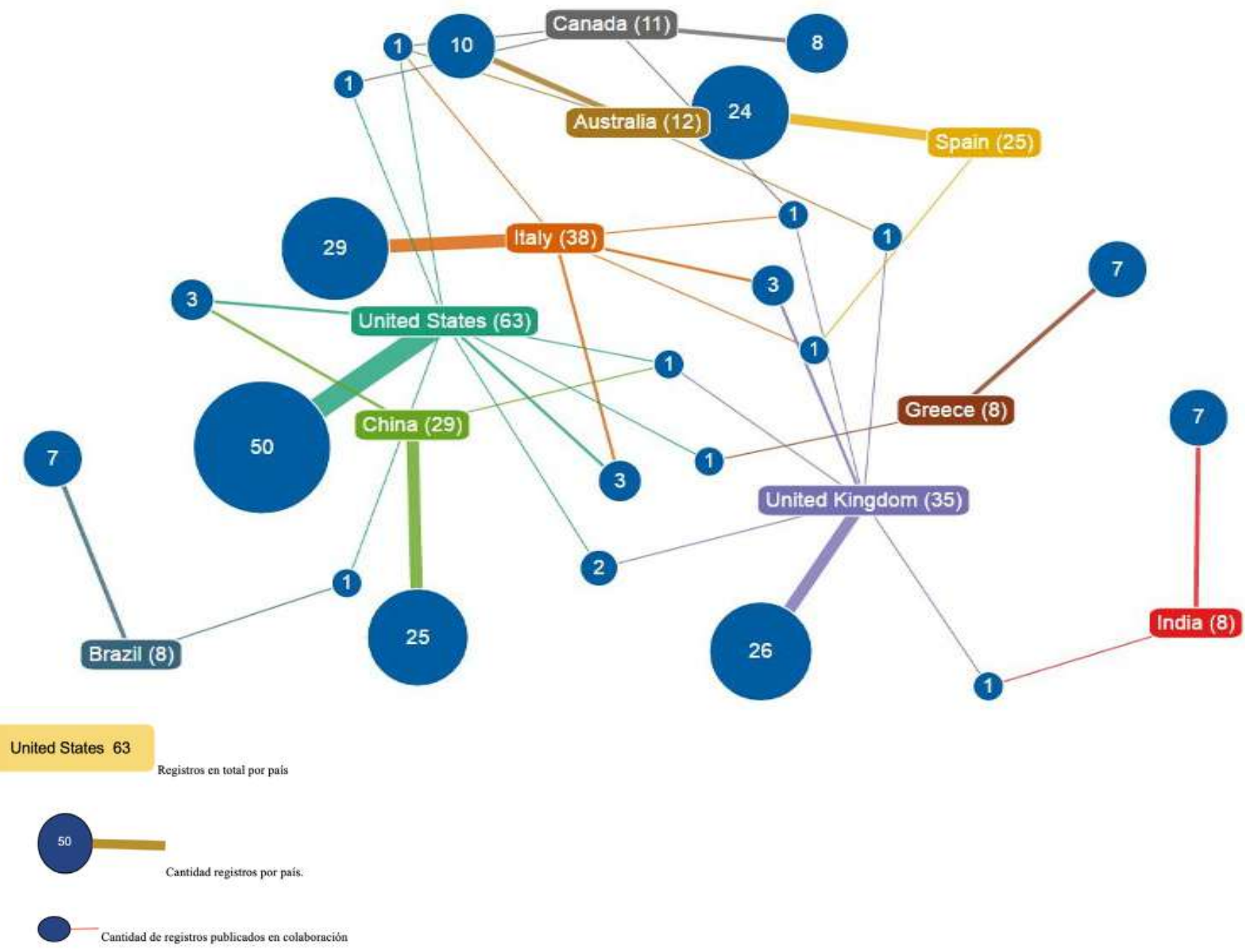

Figura 4. Clasificación de los diez países con mayor producción científica y la colaboración entre ellos.

Fuente: creación propia a partir de Vantage Point, fusión de registros de WoS y Scopus, porcentaje de cubrimiento 96 \%. 
Tabla 3. Porcentaje de colaboración entre países

\begin{tabular}{|c|c|c|c|c|}
\hline Ranking & País & Producción científica por país & Colaboración & Porcentaje de participación \\
\hline 1 & Estados Unidos & 50 & 13 & $27 \%$ \\
\hline 2 & Italia & 29 & 9 & $16 \%$ \\
\hline 3 & Reino Unido & 26 & 9 & $15 \%$ \\
\hline 4 & China & 25 & 4 & $12 \%$ \\
\hline 5 & España & 24 & 1 & $11 \%$ \\
\hline 6 & Australia & 10 & 2 & $5 \%$ \\
\hline 7 & Canadá & 8 & 3 & $5 \%$ \\
\hline 8 & Brasil & 7 & 1 & $3 \%$ \\
\hline 9 & Grecia & 7 & 1 & $3 \%$ \\
\hline 10 & India & 7 & 1 & $3 \%$ \\
\hline
\end{tabular}

Fuente: elaboración propia.

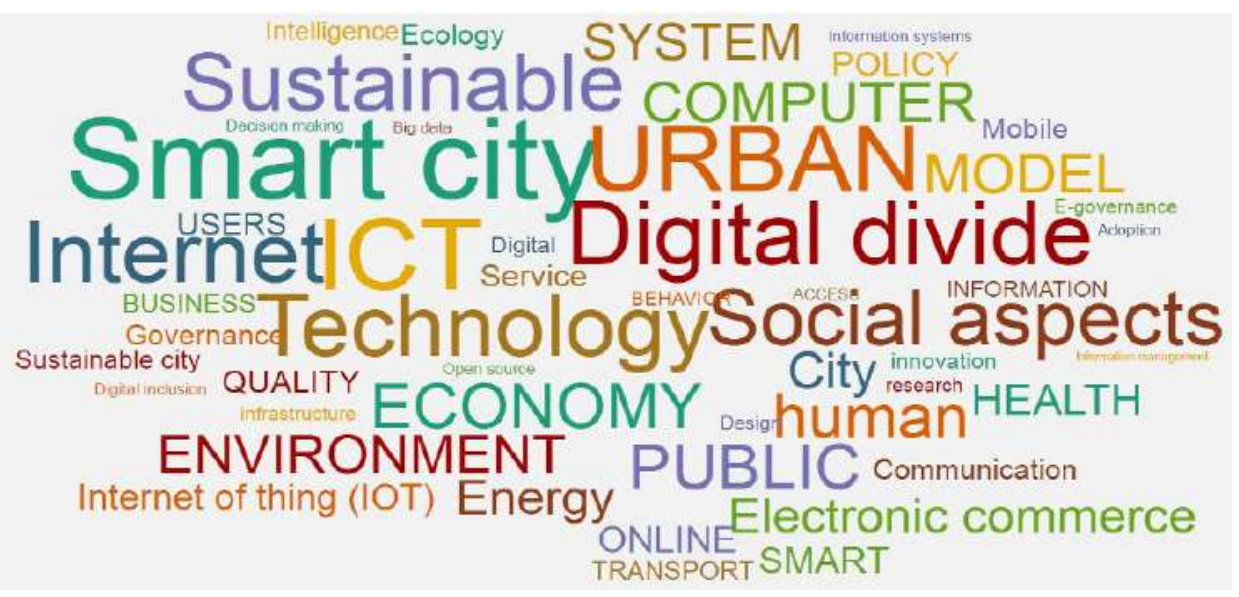

Figura 5. Palabras claves de autor y de editorial indexadas que más se mencionan en los registros.

Fuente: creación propia a partir de Vantage Point, fusión de registros de WoS y Scopus. Porcentaje de cubrimiento 92 \%. Las palabras que aparecen en un tamaño más grande son las más mencionadas en los registros.

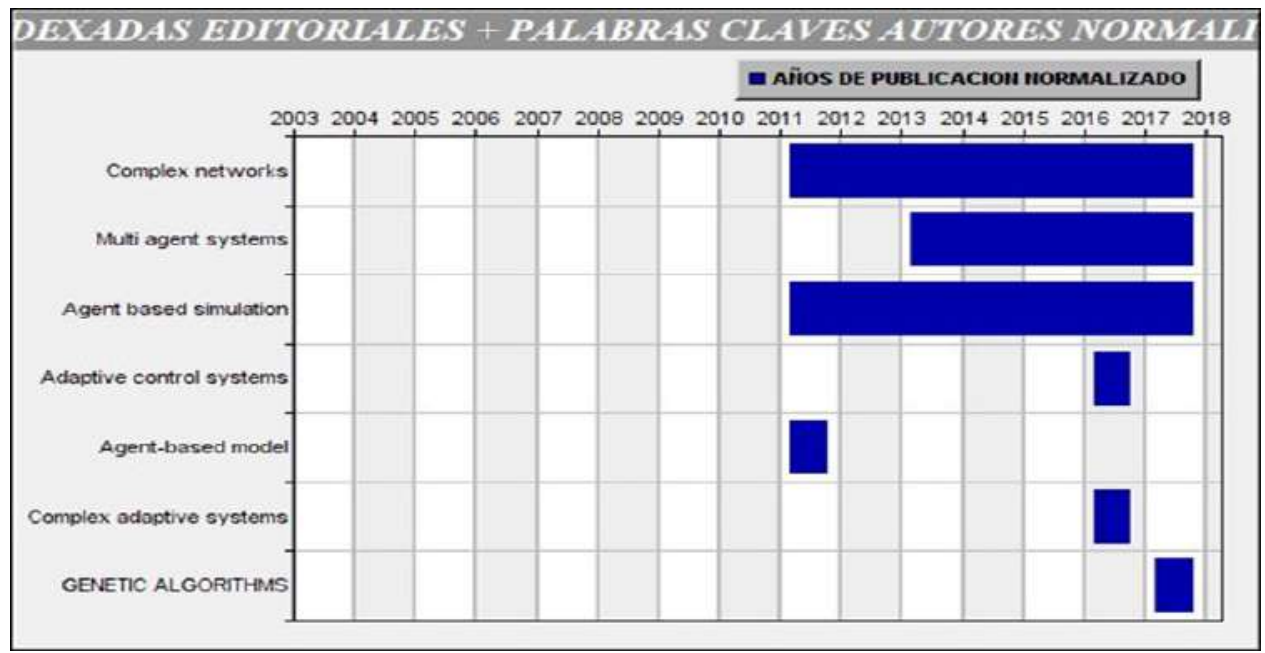

Figura 6. Herramientas de modelamiento y simulación más utilizadas.

Fuente: creación propia a partir de Vantage Point, fusión de registros de Web of Science y Scopus. Porcentaje de cubrimiento $92 \%$. Las palabras que aparecen en un tamaño más grande son las más mencionadas en los registros. 


\section{Conclusiones}

Los gráficos presentados en este artículo son el resultado de la aplicación de la metodología para vigilancia tecnológica basada en la Norma AENOR: NORMA UNE 166006 (2011) y un acercamiento exploratorio del software para minería de datos Vantage Point. Para realizar este análisis, primero se realizó un proceso de normalización y limpieza de los datos exportados de las bases de datos Scopus y WoS; este proceso fue complejo ya que se fusionaron los resultados de ambas bases de datos académicas para lograr una mayor cobertura en los resultados. En este artículo se han presentado indicadores bibliométricos de producción científica y colaboración generados a partir de la limpieza y análisis de los metadatos exportados de las bases de datos Scopus y WoS e importados a la herramienta de minería de datos Vantage Point a través de la elaboración de una estrategia de búsqueda.

Entre los resultados del proceso que cabe resaltar se menciona que el campo del modelamiento y simulación de fenómenos sociotecnológicos es un campo que se ha venido expandiendo en el campo científico, así como las diferentes líneas, instituciones e investigadores que se interesan en este tipo de fenómenos. De igual forma, se ha identificado que este campo está abierto a la investigación que desde Colombia y Latinoamérica se pueda hacer, ya que hasta el momento es nula la investigación que se ha realizado desde estas regiones. Con el proceso de revisión sistemática de literatura y minería de datos se ha confirmado que las herramientas más utilizadas para realizar procesos de modelamiento y simulación de fenómenos sociotecnológicos son las redes complejas y la simulación basada en agentes, aspecto que concuerda con las tendencias en temas de simulación.

\section{Referencias}

1. AENOR (2011). AENOR: Norma UNE 166006:201l, https://www.une.org/encuentra-tu-norma/busca-tunorma/norma?c=N0046930

2. Bider, Ilia; Otto, Henning (2015). Modeling a global software development project as a complex socio-technical system to facilitate risk management and improve the project structure. En Proceedings - 2015 IEEE 10th International Conference on Global Software Engineering, ICGSE 2015 (pp. 1-12).
Institute of Electrical and Electronics Engineers Inc. https://doi.org/10.1109/ICGSE.2015.13

3. Davidsson, Paul (2000). Multi agent based simulation: Beyond social simulation. 2nd International Workshop on MultiAgent-Based Simulation-Revised and Additional Papers (MABS '00), 97-107. Berlin, Heidelberg: Springer-Verlag.

4. Dignum, Virginia; Tan, Yao-Hua (2011). Multi agent simulation for control and autonomy in complex socio-technical systems. En 2011 International Conference on Networking, Sensing and Control, ICNSC 2011 (pp. 62-67). Delft. https://doi.org/10.1109/ ICNSC.2011.5874935

5. EcuRed (2017). Simulación (Informática). https://www. ecured.cu/Simulaci\%C3\%B3n_(Inform\%C3\%Altica)

6. Fabiano-Dalpiaz, Paolo; Mylopoulos, John (2011). Adaptive socio-technical systems: A requirementsbased approach. Requirements Engineering 18, 1-24. https:// link.springer.com/article/10.1007/s00766-011-0132-1

7. Ferscha, Alois; Zia, Kashif; Riener, Andreas; Sharpanskykh, Alexei (2011). Potential of social modelling in socio-technical systems. En Procedia Computer Science (vol. 7, pp. 235-237). Budapest. https:// doi.org/10.1016/j.procs.2011.09.051

8. Galvis, Ernesto; Sánchez, Marcela (2014). Revisión Sistemática de literatura sobre procesos de gestión de conocimiento. Revista Gerencia Tecnológica Informática, 13(37), 45-67.

9. Georgas, John; Sarma, Anita (2011). STCML: An extensible XML-based language for socio-technical modeling. En Proceedings - International Conference on Software Engineering (pp. 6l-64). Waikiki, Honolulu, HI. https://doi.org/10.1145/1984642.1984657

10. Hassannezhad, Mohammad; Cantamessa, Marco; Montagna, Francesca (2015). Actor-based signposting: A modeling tool to improve the socio-technical design processes. En Proceedings of the International Conference on Engineering Design, ICED (vol. 3, pp. 165-174). Design Society. https://www.scopus.com/inward/record.uri?eid=2-s2.0$84979917146 \&$ partnerID=40\&md5=147e9e0b58860177e 6 ecc25c61624f19

11. Lee, Dong-Ah (2006). Socio-technical Systems. Konkut University. http://dslab.konkuk.ac.kr/Class/2013/13SE/ ClassB/lecturenotes/ch2.pdf

12. Ropohl, Günter (1999). Phylosophy Documentation Centar. Techné: Research in Philosophy and Technology 4(3), 186-194. https://philpapers.org/rec/ROPPOS 
13. Sharpanskykh, Alexei (2011). Agent-based modeling and analysis of socio-technical systems. Cybernetics and Systems, 42(5), 308-323. https://doi.org/10.1080/0196972 2.2011 .595332

14. Sánchez-Meca, Julio (2010). Cómo realizar una revisión sistemática y un meta-análisis. Aula abierta, 38(2), 53-64. https://www.um.es/metaanalysis/pdf/5030.pdf

15. UTADEO (2017). ¿Qué es modelado y simulación (M\&S)? http://www.utadeo.edu.co/es/link/maestriaen-modelado-y-simulacion-mms/26106/layout-1/quees-modelado-y-simulacion-ms 